\title{
Handwritten Mathematical Expressions Recognition using Back Propagation Artificial Neural Network
}

\author{
Sagar Shinde \\ JSPM, Narhe Technical Campus, \\ Savitribai Phule Pune University, \\ Pune-411041, \\ Maharashtra, India.
}

\author{
Rajendra Waghulade, PhD \\ DNCVP, North Maharashtra University, \\ Jalgaon- 425001, \\ Maharashtra, India.
}

\begin{abstract}
Handwritten mathematical expressions recognition is yet challenging task due to its intricate spatial structure, tangled semantics and 2-dimensional layout of the characters. There is a still room for enhancement in recognition rate. Artificial neural network is superior to disentangle classification problems. In this paper, feedforward back propagation neural network is implemented to achieve both character recognition and mathematical structure recognition with upgrade in effective performance in addition to accuracy of the experimental results including lessen efforts. System proves its potency by recognizing expressions in analysis of math documents.
\end{abstract}

\section{Keywords}

Character recognition, Math symbol recognition, Handwritten math equations, Feed forward back propagation neural network.

\section{INTRODUCTION}

Mathematics is almost unavoidable in all fields of Science such as physics, engineering, medicine, economics also in commerce field such as accountancy. Handwritten text recognition system have achieved recently noteworthy improvement, but many issues have not be solved such as recognizing two dimensional ( $2 \mathrm{D}$ ) layout which is the case for mathematical expressions.[1][2]

In mathematical expressions, there are lots of feature, which contain enclosed relation of the multidimension, miscellanea of the characters, multifariousness of size and font of the character, symbols are placed in diagonal, horizontal or vertical directions. Therefore, recognition of mathematical symbol and expression is considered as an serious pattern recognition problem. Mathematical formula contain two dimensional information, there are two problems have to solve; symbol recognition and structure analysis. Handwritten math symbols and expressions recognition is one of the most challenging areas. It requires tangled task of symbol segmentation, recognition, structure investigation, context investigation and total probable evaluation. Math notation, symbols, expressions are widely useful in real world practical difficulties includes, document verification, mailing address interpretation, bank checking processing, signature verification, blind math applications etc.

For dealing with mathematical formulas, the system should be made to execute both character and mathematical structure recognition based on the integration and bilocation of characters. A handwritten input has affable nature as well as high expression prospective. It is look forward to crenellate expeditious input methods for intricate equations such as mathematical equations which incorporate vertical upended placed symbols obtain from particular structures in opposition with plain text where symbols are adjust according to horizontal line. Some difficulties still exist in recognition of mathematical expressions including expected recognition rate if more noise in image of expression and different problems to correct the recognition errors in recognition. Mathematical equation require numerous tag instructions in LATEX. A lot of actions of the package are intricate in mathML so handwritten mode is better natural way of inserting mathematical notation into computers. Neural network is one of the most accurate learning methods to approximate real valued, discrete valued and vector valued functions. neural network based on multiple layer perceptron with back propagation algorithm shows good performance and have been successfully applied to the character recognition systems with small error margins.

\section{HISTORICAL BACKGROUND}

The indoctrination which integrates structural information and semantic rule based on statistic in the post processing, boost the system of the mathematical formulas recognition at great extent and upgrade accuracy of the results [3]. The denouement for mathematical expression recognition can be subdivided into two main problems segmentation and recognition, investigation anagoges of symbols spacial arrangement [4]. When scientific document is recognized by optical character recognition software, the expression on it will be saved as an image which cannot be reused. So question is arise that how to recognize mathematical expression automatically becomes most important and challenging problem in pattern recognition [5. Neural network is efficacious and magnificent methods to approximate real-valued, discrete valued and vector valued functions. It has been mostly useful in classification and regression difficulties such as handwritten characters, symbols and digits recognition [6].

Offline character recognition is relatively complicated than online character recognition as different person have different kind of handwriting mode and also the characters are extracted from the documents of heterogeneous intensity and background [7]. weighty class of feed forward neural network is the back propagation neural network. System is promising, using it clarification can be made by consecrating global information and interpretation can be reached by admissible heuristic function [8]

Validation of segmentation point is integral part. It is developed to remove invalid segmentation points and 
retain the valid points in order to increase accuracy of the overall segmentation [9]. Technique is depend on contour feature that partitioning paths can be grade constructed on contour feature and the ratio of width and height and then touching characters are segmented [10]. The method of contour approach for extracting symbols from the image representing mathematical expression and improve the result by proposing complete segmentation method which treats both of sub-segmentation upon-segmentation problems [11].

Actually artificial neural network is a good to solve classification problems. ANN can perform computations at higher rate compared to classical techniques because of its parallel nature[12]. During processed the data, training stage and classification stage are the two important stages. It is known to be better in terms of speed and recognition rate. The designed artificial neural network based on feed forward back propagation algorithm. It shows good power and performance and has been successfully applied to mathematical expressions recognition with small error margins. It is a multilayer feed forward network using gradient descent based learning rule usually known as a back propagation rule. It indulge a computationally competent method for varying the weights in feed forward network with differentiable activation function units, to grasp a training set of input output examples. our supervised feed forward back propagation neural network consists of one input layer, two hidden layer and output layer. In feed-forward network, training set comprises sets of input values together with the corresponding sets desired output values. Learning process involves adjusting the values of parameters to minimize the value of error functions. Back propagation learning algorithm is most popular supervised learning rule for performing pattern classification task. Supervised learning method is used to trained the network and total square error of the output calculated by net is minimizes by gradient descent method usually known as generalized delta learning rule. The network is train to accomplish balance between to react precisely to the input characters that are used for training and the ability to produce best responses to the input that were matched.

The basic idea in the back propagation neural network is that the error is propagated backward to previous layers so that gradient descent can be applied. Multilayer perceptron network trained by error back propagation algorithm is superior in recognition accuracy.

In proposed system after initialize the neural network, train it with training set. In each and every training interval all the training characters, symbols, digits and operators go through network and get real network output. The network error is obtained by examining actual network and target output. Network errors are then applied in updating all network weights. After training and validating process, believe that neural network has been well trained and can be used for character classification. Before training weights in each network were independently set to random initial values. To be a successful classification it must be preceded by a training stage [13]. An experimental result shows the improvement in

mathematical expression recognition with effectiveness and accuracy.

\section{SYSTEM ARCHITECTURE}

The system architecture of handwritten mathematical equations recognition using artificial neural network is as shown in figure 1 . Basically there are two stages, training stage and testing stage. It involves preprocessing, segmentation, feature extraction, training of classifier, testing of classifier.

Acquiring the image: First of all, we require generating digital image from paper envelope by using CCD camera or Scanner.

Preprocessing: It is executed on the scanned input image in order to render the resulting image more suitable for further operations. It includes noise removal, binarization, skew correction, enhancing the contrast and identifying regions likely to contain the mathematical expression. In pre-processed input image is segmented into isolated characters by allocating number to each character using labeling process. Labeling provides information about number of characters in the image.

Segmentation: Image is decomposed into sub-images of individual characters. We extract from the image that part of it that contains only mathematical equation. The preprocess image is divided into lines words and characters. To separate the text lines, line segmentation is used.

Word segmentation is providing the space between words and character segmentation gives spacing between the characters. Lines are segmented using row histogram. From each row, words are extracted using column histogram and finally characters are extracted from words. Each character is resized into $\mathrm{m} \times \mathrm{n}$ pixels towards the training network. Actually the accuracy of the final result is mostly depends on the accuracy of segmentation.

Feature Extraction: It improves the recognition rate. Average pixel method involves reduction of the size of the each character to a normalized dimension average value of the pixels of each area is computed. Ratio between initial width and height of character is also computed and included in feature vector. In normalized contour analysis, number of intersections between layout of the character and vertical - horizontal lines also taken into account. Finally discriminant analysis is executed to abolish redundancy and noise. Statistical feature is based on the probability theory and hypothesis. Structural feature describes the geometrical and topological properties of character.

Classification: There are various classifying methods are found such as template matching which is slow and time consuming. Structural recognition are not widely used where extract structural primitives and use them by

comparing with the training data. Artificial neural network better in terms of speed and recognition rate. The standard multilayer perceptron is consists of one input layer, number of hidden layer and output layer. For pattern classification task most common neural network is feed forward network includes multilayer perceptron, organized into layers and there is a unidirectional connections between the layers. Feed forward back propagation neural network includes multilayer perceptron, organized into layers and there is a unidirectional connection between the layers. 
Back propagation learning algorithm is most powerful, effective and popular supervised learning rule for Performing pattern classification task. Learning process involves adjusting the value of parameters to minimize the value of error function. Moreover, as the normalized
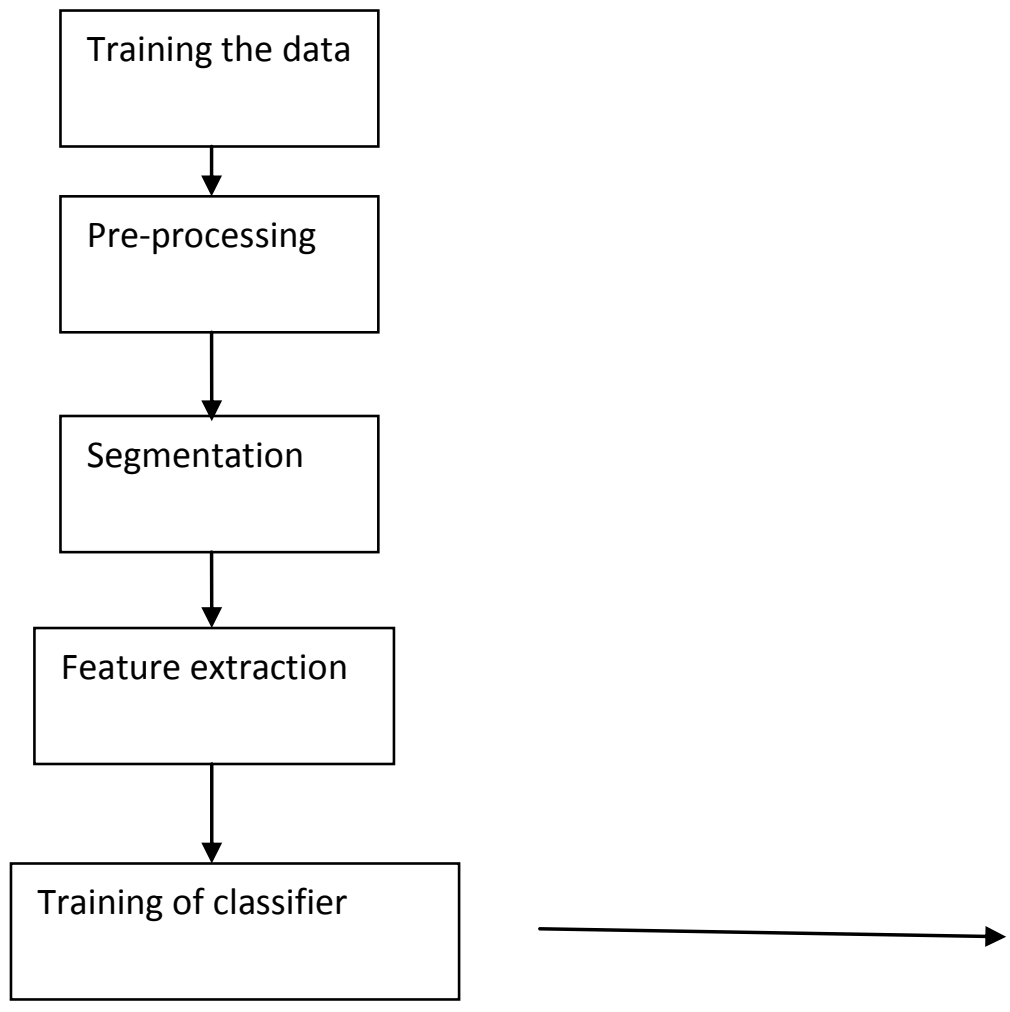

contour analysis (NCA) method and Adaptive Learning rule has appeared to be more efficient are applied in Combination with average pixel method to improve recognition rate significantly. The multilayer feed forward neural network used to recognize equations
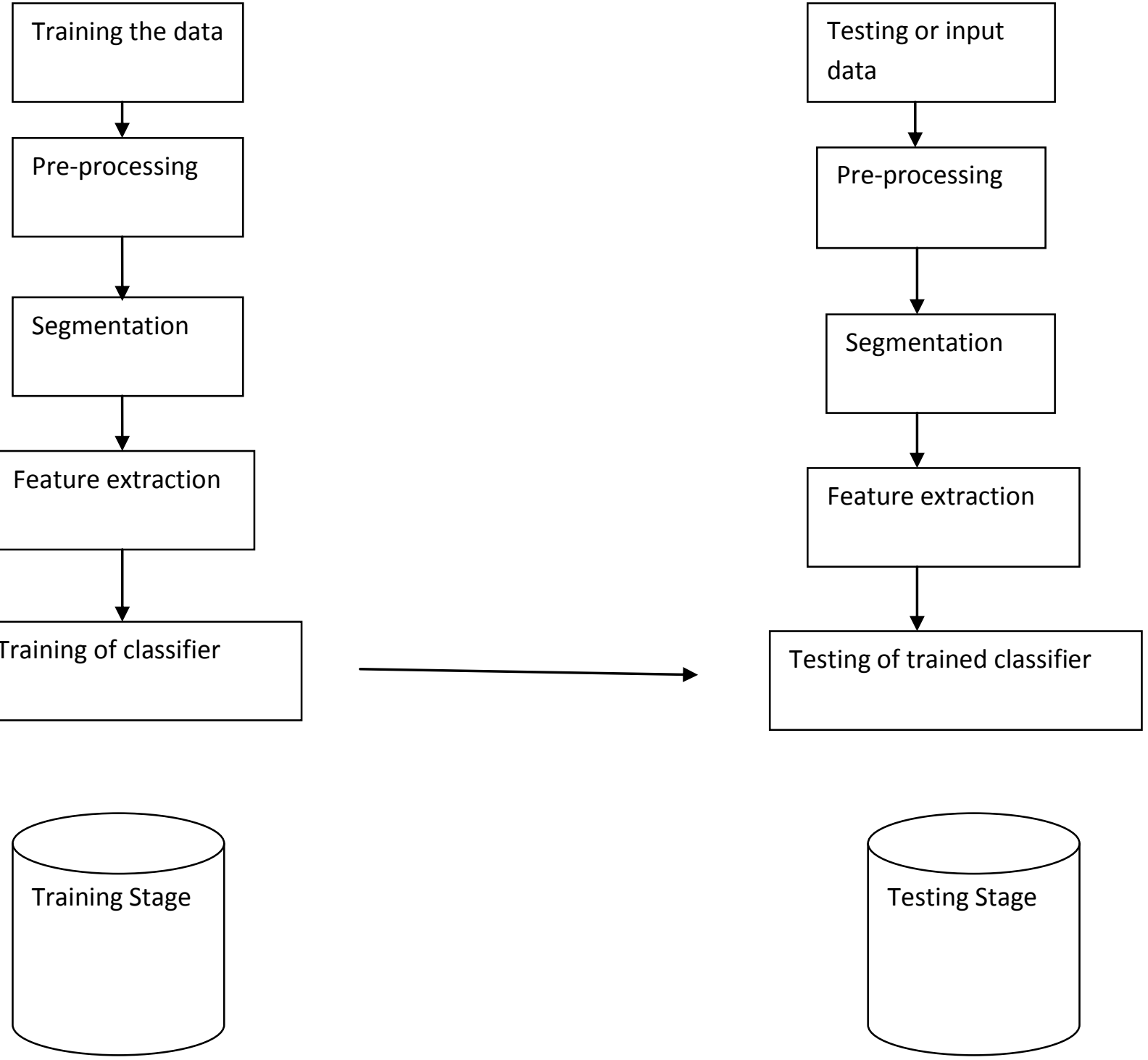

Fig 1: Block diagram of handwritten mathematical equation recognition system

\section{METHODOLOGY}

Feed forward back propagation neural network technique is used for recognition of handwritten mathematical equations by considering rectangle with respect to position, edge color, line width then finding region properties bounding box and centroid for every component. Declare the input alphabets and target for the network. Create a feed forward back propagation network with inputs and transfer function. Using logsig T. F. for input and hidden layer, traingdx T. F. for output layer then train and test the network. Network training function is gradient descent with momentum.

\section{SIMULATION RESULTS}

In proposed system, recognition of straight line equation $\mathrm{y}=$ $\mathrm{mx}+\mathrm{c}$ has done with better accuracy and recognition rate. In this equation $\mathrm{m}$ is the gradient and $\mathrm{y}=\mathrm{c}$ is the value where the line cuts the y axis.

This no. $\mathrm{c}$ is called the intercept on the y axis. The centroid, bounding box and recognition of the handwritten straight line equation is as shown below in figure 2 and figure 3 respectively. 


\section{$y=m x: c$}

Figure 2: Centroid \& bounding box

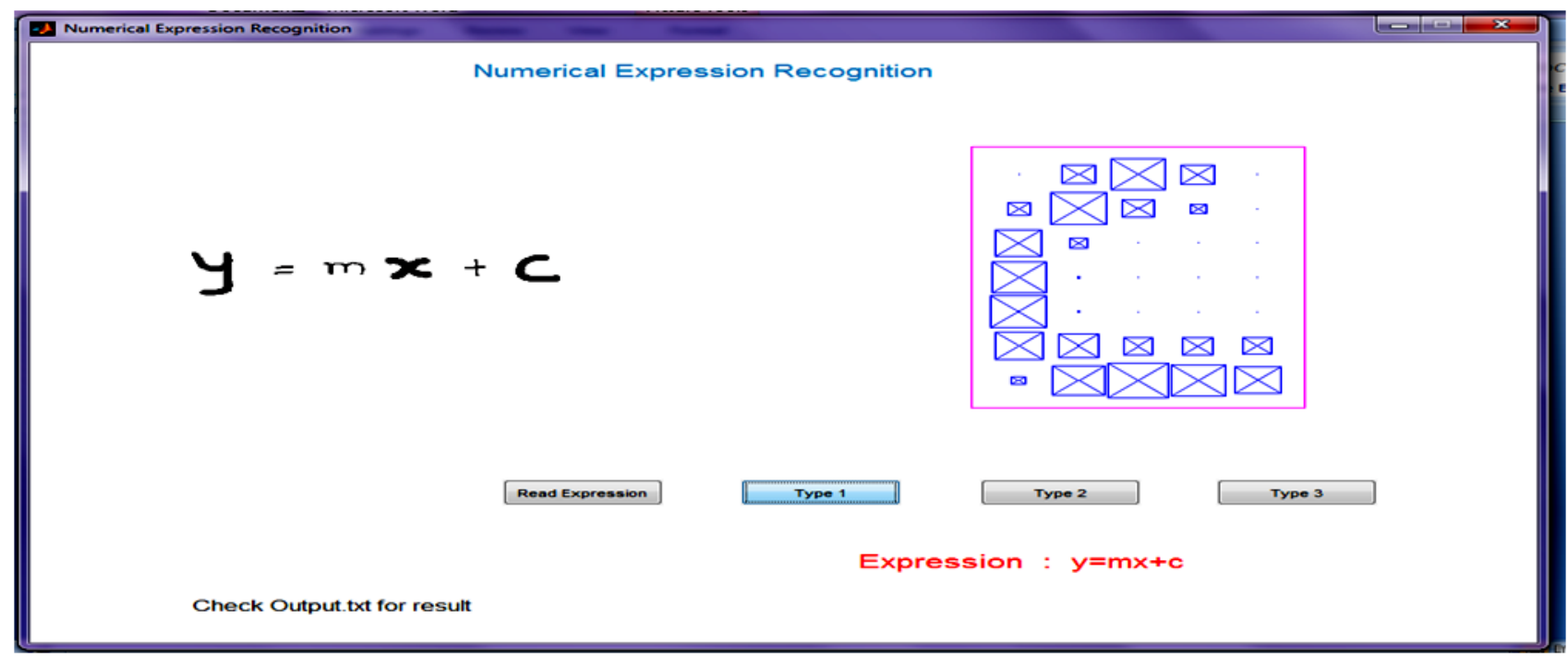

Figure 3: Handwritten straight line equation recognition.

The quadratic function is a second order polynomial function $f(x)=a x^{\wedge} 2+b x+c$. The solutions to the quadratic equation are the roots of quadratic function, that are the intersection points of the quadratic function graph with $\mathrm{x}$ axis, when $\mathrm{f}(\mathrm{x}$ ) $=0$. When there are two when there are 2 intersection points of the graph with the $\mathrm{x}$-axis, there are 2 solutions to the quadratic equation. When there is 1 intersection point of the graph with the $\mathrm{x}$-axis, there is 1 solution to the quadratic equation.
When there are no intersection points of the graph with the $\mathrm{x}$ axis, get not real solutions (or 2 complex solutions). The Centroid of quadratic equation is as shown below.

\section{$a x^{2}+b x+a c=0$}

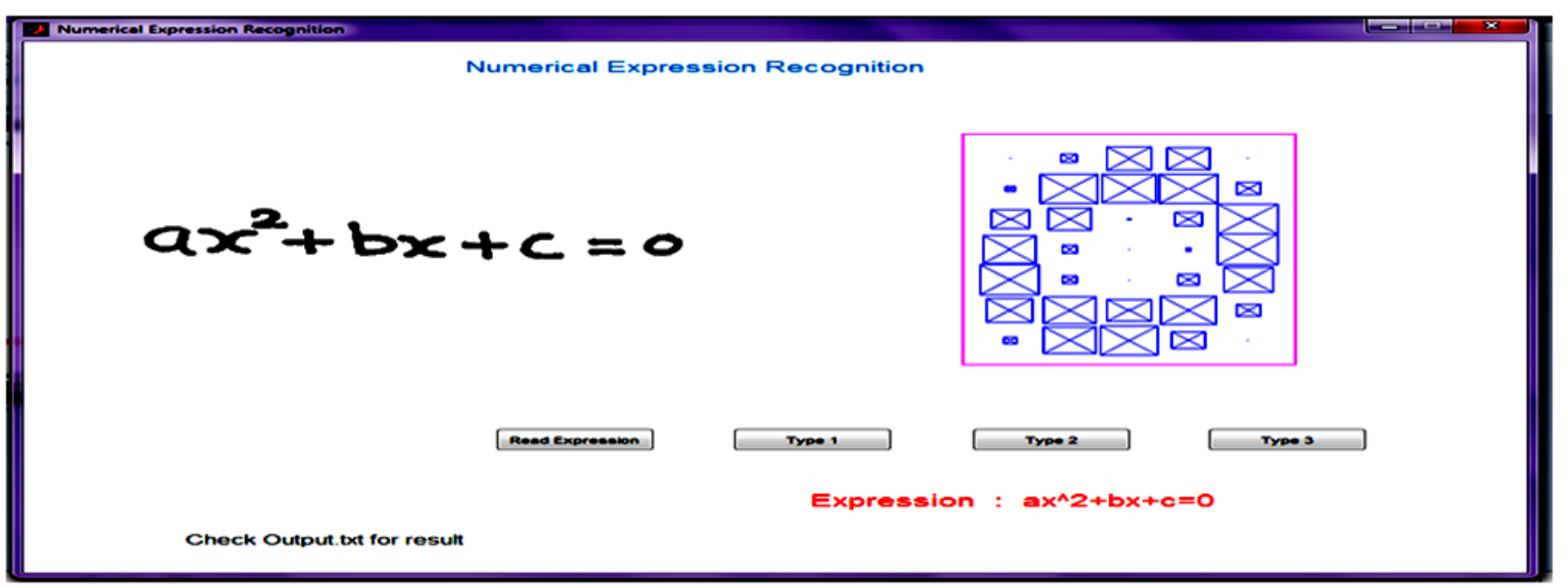

Figure 5: Handwritten Quadratic equation recognition 


\section{Neural Network Training (nntraintool)}

Neural Network
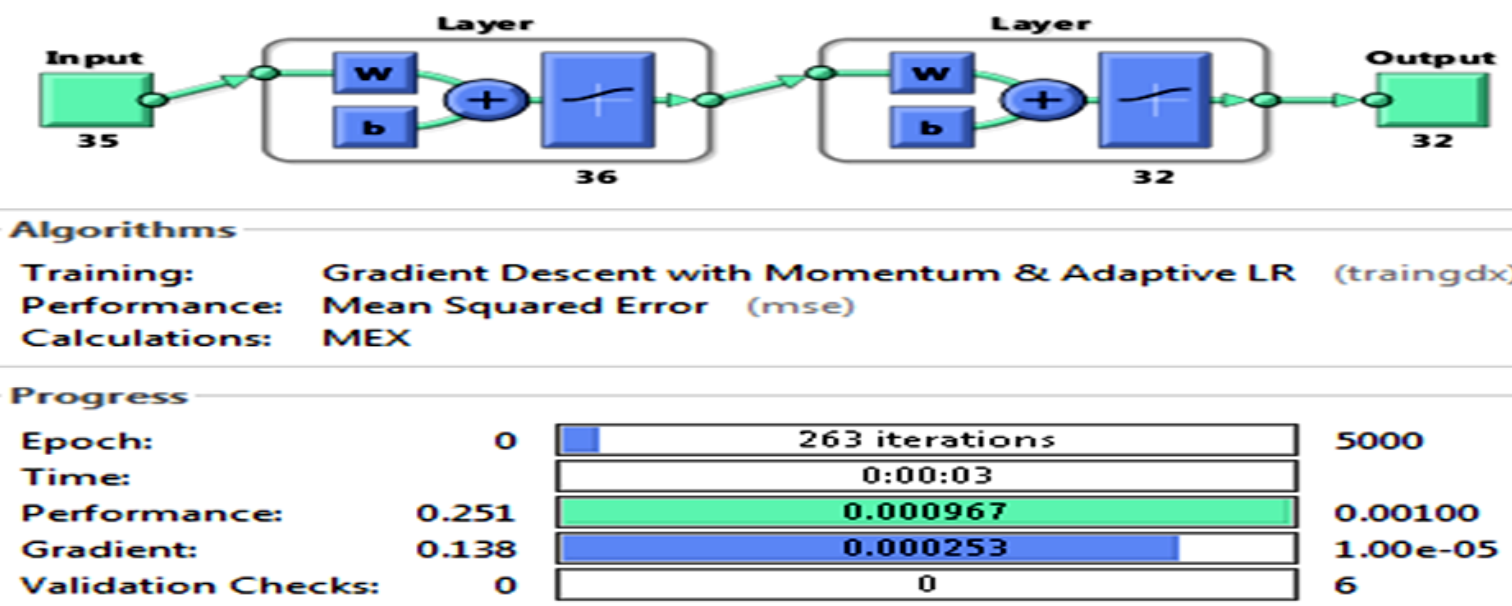

5000

\section{Plots \\ Performance (plotperform) \\ Training State (plottrainstate) \\ Regression (plotregression)}

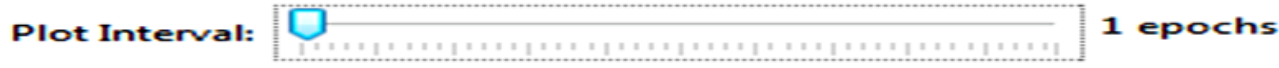

\section{Opening Training State Plot}

Figure 6: Neural network training for handwritten mathematical equations recognition

Figure 6 shows network parameters adjusted for the training purpose. The neural network trained using gradient descent with momentum and adaptive learning. Neural Network developed using one input layer, two hidden layers and one output layer. By repeatedly presenting data set to the network, performance of network is increase. An optimum selection of number of neurons in the hidden layer gives good performance.

Table 1. Neural network training parameters.

\begin{tabular}{|c|c|c|}
\hline Sl. No. & Parameter & Value/ Type \\
\hline 1 & Network Training Function & Gradient Descent with momentum \\
\hline 2 & Learning Rule & Adaptive LR \\
\hline 3 & Network performance Function & Mean Squared Error ( MSE ) \\
\hline 4 & Number of Epochs & 263 \\
\hline 5 & Number of Iteration & 0.00100 \\
\hline 6 & Performance & $1.00 \mathrm{e}-05$ \\
\hline 7 & Gradient & i.e 0.00025321 at Epoch 263 \\
\hline 8 & Learning Rate & 3739.2599 at Epoch 263 \\
\hline
\end{tabular}

Table 1 shows various neural network training parameters. Performance of the developed system is measured and system accuracy, learning rate is calculated. More number of hidden layer neurons lead to overtraining

\section{CONCLUSIONS}

This system presented an approach to recognize handwritten mathematical equations such as straight line equation and quadratic equation with improvement in recognition rate, performance, processing time and accuracy. Online entry of handwritten mathematics into electronic Documents will become more powerful and user- friendly. The classifiers have been designed to capture wide variations in shape and size of the large number of symbols that occur in writing math symbol and applications. 
Simulation results show propitious results. Centroid and bounding box are the key features that are extracted from each character and uprightness of this system is achieve using back propagation neural network for the recognition of mathematical equations.

\section{REFERENCES}

[1] Ahmad-Montaser Awal, Harold Mouchere, Christian Viard-Gaudin, 2009, "Towards Handwritten Mathematical Expressions Recognition", IEEE 10th International Conference on Document Analysis and Recognition, ICDAR 2009, Jul 2009, Barcelone, Spain. Pp.1046-1050, 2009.

[2] Dov Dori, David Doermann, Robert Haralick, Ihsin Phillips, Mitchell Buchman, David Ross. 1996, “ The representation of document structure: A generic objectprocess analysis", Handbook on optical character recognition and document image analysis, World scientific publishing company, 1996.

[3] Wen-jiao Wang, Lei Han, Tian-yu Chen, Xue-dong Tian, 2007. "The Post-Processing for Mathematical Expressions Oriented on Integrative Rectification" ieeecomputersociety. pp: 894-897.

[4] DOROTHEA BLOSTEIN $\dagger$ and ANN GRBAVEC,1996, “ Recognition of mathematical notation", Handbook on optical character recognition and document image analysis, World scientific publishing company, 1996

[5] Ming-Hu Ha , Xue-dong Tian, Na Li, 2006, "Structural Analysis of Printed Mathematical Expressions Based on Combined Strategy " International Conference on Machine Learning and Cybernetics, Cybernetics, Dalian, China. pp 3354 - 3358, ISBN: 1-4244-0061-9."

[6] Tom M. Mitchell, 1997, International edit “ Machine Learning” McGraw-Hill Science/Engineering/Math ISBN:0070428077.

[7] Anurag Bhardwaj, Faisal Farooq, Huaigu Cao, Venu Govindaraju. 2008," Topic based language models for
OCR correction", Proceedings of the second workshop on Analytics for noisy unstructured text data, New York, NY, USA, Pages 107-112, ISBN: 978-1-60558-196-5

[8] Dae Hwan Kim, Kim, J.H.. 2010, “Top-Down Search with Bottom-Up Evidence for Recognizing Handwritten Mathematical Expressions", IEEE International Conference on Frontiers in Handwriting Recognition, Kolkata, pp 507 - 512, ISBN: 978-1-4244-8353-2.

[9] Al Hamad H.A, 8-10 Oct. 2013, "Use an efficient neural network to improve the Arabic handwriting recognition" , IEEE International Conference on Signal and Image Processing Applications, Melaka, pp 269 - 274, ISBN: 978-1-4799-0267-5.

[10] Xuedong Tian, Yan Zhang. July 30 2007-Aug.1 2007, "Segmentation of Touching Characters in Mathematical Expressions Using Contour Feature Technique “, IEEE Eighth ACIS International Conference on Software Engineering, Artificial Intelligence, Networking, and Parallel/Distributed Computing, 2007. (SNPD- 2007), Qingdao, pp- 206 - 209, ISBN:978-0-7695-2909-7.

[11] Jakjoud, W, Lazrek, A., 7-9 April 2011, "Segmentation method of offline mathematical symbols", IEEE International Conference on Multimedia Computing and Systems (ICMCS), Ouarzazate, pp. 1 - 7, ISBN: 978-161284-730-6.

[12] S. N. Sivanandam, S Sumathi, S. N Deepa , “ Introduction to neural network using MATLAB 6.0", Tata Mcgraw- Hill Publishing company Limited,2006, ISBN : 0-07-059112-1.

[13] N. VenkateswaraRao, Dr. A. Srikrishna, Dr. B. RaveendraBabu, G. Rama Mohan Babu, October 2011, “ An efficient feature extraction and classification of handwritten digits using neural networks", International Journal of Computer Science, Engineering and Applications (IJCSEA) Vol.1, No.5, DOI : 10.5121/ijcsea.2011.1505 